\title{
In-situ microfibrillar PP-PA6 composites: rheological, morphological and mechanical properties
}

\author{
ALI SAFAEI, MAHMOOD MASOOMI* and SEYED MOHAMMAD REZA RAZAVI \\ Department of Chemical Engineering, Isfahan University of Technology, Isfahan 84156-83111, Iran \\ *Author for correspondence (mmasoomi@cc.iut.ac.ir)
}

MS received 15 June 2016; accepted 21 November 2016; published online 23 August 2017

\begin{abstract}
In this study, the rheology, morphology and mechanical properties of microfibrillar composites based on polypropylene reinforced with polyamide 6 (PA6) fibres have been investigated. Influence of different factors such as the PA6 composition and drawing ratio on the output of the extruder, and the compatibilizer composition on the properties of these composites, were investigated. The results of rheometric mechanical spectrometer rheological measurements and melt flow index (MFI) showed that the storage modulus, loss modulus and complex viscosity of the microfibrillar composites are directly related to the composition of PA6. It was also shown that the presence of compatibilizer was the main factor that led to decrease of some properties including the storage modulus, loss modulus and complex viscosity and increase in the MFI. The results of mechanical tests on samples prepared with different drawing ratios showed that by increasing the drawing ratio from 3 to 5, the mechanical properties increase, while increasing draw ratio from 5 to 8 reduces the mechanical properties. With the increase in the composition of the polyamide phase, the tensile strength of microfibrillar composite compatible with polypropylene-grafted-maleic anhydride initially increased; however, the tensile strength decreased with a high composition of polyamide phase. Scanning electron microscope images also revealed that the presence of the compatibilizer in the microfibrillar composites leads to decrease in the number of undeformed drops, while it reduces the micro-fibres diameter, which greatly improved the final properties of the composites.
\end{abstract}

Keywords. In-situ microfibrillar composite; rheology; morphology; compatibilizer.

\section{Introduction}

Fibres composites are one of the major types of composites, which are distinguished with their higher strength and stiffness and lower density than those of other materials with similar properties, and targeted orientation. Over the past two decades, environmental concerns, besides global desire for removing mineral materials from polymer composites, led to synthesis of new types of composites, known as 'polymer-polymer' composites. Both the matrix phase and reinforced phase in these composites are selected from the polymeric materials. The polymer-fibre polymer composites can be synthesized using two approaches. In the first method, pre-prepared polymer fibres are added to the polymer matrix. In The second method, immiscible polymer blends are prepared and then polymer fibres are synthesized in situ in the polymer matrix. The composites synthesized by the second method are known as microfibrillar composites $[1,2]$.

Polyolefin microfibrillar composites possess some prominent properties that have attracted the attention of researchers, including the nonnecessity of mixing the fibres and the polymer matrix; micro-fibres are synthesized in the polyolefin matrix, in situ; the synthesized micro-fibre is completely dispersed within the matrix and fibre distribution in the matrix does not require the benefit of drop-matrix morphology in the prepared polymer blend; moreover, the composite has low density, easy processing and is of reasonable price [3].

The various applications of microfibrillar composites have been studied by some researchers. These studies have discussed various parameters of microfibrillar composites such as the reinforced and matrix phase materials, composition of each phase, rheological properties, mechanical properties, morphology and the compatibilizer effect on the final properties [4-14]. All studies carried out proved that the application of microfibrillar composites led to various and favourable properties in the final product.

Given the importance of these structures, we also aim to investigate the rheology and morphology of microfibrillar composites based on polypropylene (PP) reinforced with polyamide 6 (PA6) fibres. The main focus of this study is to investigate the properties of microfibrillar composites by altering factors such as the composition of PA6 (reinforced phase), and the effect of different drawing ratios on and the composition of the applied compatibilizer on the output of the extruder, and evaluate the morphological type of reinforced phase based on the properties of these composites. 


\section{Materials and methods}

\subsection{Materials}

The materials used in this study to prepare microfibrillar composites include PA6 (manufactured by BASF, Germany, B40 $\mathrm{L}$ grade as the reinforced phase, melting point $=220^{\circ} \mathrm{C}$ ), $\mathrm{PP}$ (grade C30S Maroon Petrochemicals as the matrix, melt flow index $(\mathrm{MFI})=6 \mathrm{~g} / 10 \mathrm{~min}$ ), Irganox powder (produced by the Swiss company Ciba as the antioxidant) and polypropylenegrafted-maleic anhydride (PP-g-MA, produced by Honam Petrochemical Company in South Korea, branded as CM$1120 \mathrm{H}$, as the compatibilizer).

\subsection{Microfibrillar composite preparation}

A single-screw extruder (Plasticorder Lab-Station) with a slit die of dimensions of $2 \times 20 \mathrm{~mm}$ (Brabender Co., Germany) was used to melt the polymers and prepare the blends. A drawing device was employed to apply various draw forces to the strip-out of the die extruder. Finally, a compression moulding machine was used to mould the prepared microfibrillar composites.

2.2a Sample preparation method: The process of synthesizing the micro-fibre composites includes three stages: melt blending of two immiscible polymers, creating fibre morphology and finally the process of forming a microfibrillar composite. In the first stage, PP, PA6 and PP-g-MA were placed in a vacuum oven for $12 \mathrm{~h}$ at $80^{\circ} \mathrm{C}$ to remove the moisture in the materials. Afterwards, as shown in table 1, materials

Table 1. Coding the prepared samples.

\begin{tabular}{lcccc}
\hline Code & PP & PA6 & PP-g-MAH & Draw ratio \\
\hline PP & 100 & - & - & - \\
PA6 & - & 100 & - & - \\
PP-g-MAH & - & - & 100 & - \\
10PA-0C-D3 & 90 & 10 & 0 & 3 \\
5PA-5C-D3 & 90 & 5 & 5 & 3 \\
10PA-5C-D3 & 85 & 10 & 5 & 3 \\
15PA-5C-D3 & 80 & 15 & 5 & 3 \\
10PA-0C-D5 & 90 & 10 & 0 & 5 \\
5PA-5C-D5 & 90 & 5 & 5 & 5 \\
10PA-5C-D5 & 85 & 10 & 5 & 5 \\
15PA-5C-D5 & 80 & 15 & 5 & 5 \\
10PA-0C-D8 & 90 & 10 & 0 & 8 \\
5PA-5C-D8 & 90 & 5 & 5 & 8 \\
10PA-5C-D8 & 85 & 10 & 5 & 8 \\
15PA-5C-D8 & 80 & 15 & 5 & 8 \\
10PA-10C-D5 & 80 & 10 & 10 & 5 \\
10PA-0C-D0 & 90 & 10 & 0 & 0 \\
5PA-5C-D0 & 90 & 5 & 5 & 0 \\
10PA-5C-D0 & 85 & 10 & 5 & 0 \\
15PA-5C-D0 & 80 & 15 & 5 & 0 \\
\hline
\end{tabular}

were transmitted to the extruder hopper after being weighed carefully and completely mixed together. Since both polymers in the blending stage should be in the molten state, the temperature profiles $190-220-240$ and $250^{\circ} \mathrm{C}$ of the extruder with 80 RPM screw speed were used. In the second phase, the blend output from the extruder was passed through a cold water bath and placed between the rollers of the drawing device in order to create fibre morphology. Different drawing ratios of 3, 5 and 8 were applied by the drawing device to pull the plastic strip out of the extruder machine. The area for hot drawing was placed between the die extruder and entrance of the strip to the cool water bath. The tape obtained from the drawing device, which is the microfibrillar composite, was collected and cut into 2-3 cm pieces. Microfibrillar composites strips were formed by the pressure moulding machine and placed in the vacuum oven for $12 \mathrm{~h}$ at $80^{\circ} \mathrm{C}$; then they were randomly poured into the mould at a temperature of $190^{\circ} \mathrm{C}$ and pressure of $100 \mathrm{bar}$ for $4 \mathrm{~min}$, and 3-mm-thick sheets were prepared to be used for rheometric mechanical spectrometer (RMS) tests.

\section{Results and discussion}

\subsection{Rheological results}

Different tests were carried out and indices measured to study the rheology of the microfibrillar composites as a function of three parameters: (1) the composition of the reinforced phase (PA6), (2) drawing ratio and (3) the compatibilizer composition. These tests include the RMS, MFI and capillary rheometer (parallel plate rheometer with $1 \mathrm{~mm}$ distance plate and $12.487 \mathrm{~mm}$ radius, model 301, made by Anton Paar Co., Austria and capillary rheometer with $L / D=40$, made by Instron, were used for rheology tests) tests.

3.1a RMS test results: The effect of three parameters: drawing ratio, composition of the reinforced phase (PA6), and the compatibilizer composition, on the rheological properties of microfibrillar composites was investigated by RMS test.

Pure material properties The loss modulus, complex viscosity and storage modulus for PP, PA6 and pure PP-g-MA are shown in figure $1 \mathrm{a}-\mathrm{c}$.

Draw ratio effect on $R M S$ test results Figure $2 \mathrm{a}-\mathrm{c}$ shows storage modulus, loss modulus and complex viscosity of the microfibrillar composites synthesized at a drawing ratio of 3,5 and 8 . It can be seen that increase in the drawing ratio from 3 to 5 causes more restriction for the movement of the PP chain and more external energy is needed for a certain amount of deformation in the composite subsequently; therefore, the storage and loss modulus and the complex viscosity increase. However, the rheological properties reduce due to the orientation of microfibres in the direction of draw and entanglement is reduced at higher drawing ratio. 

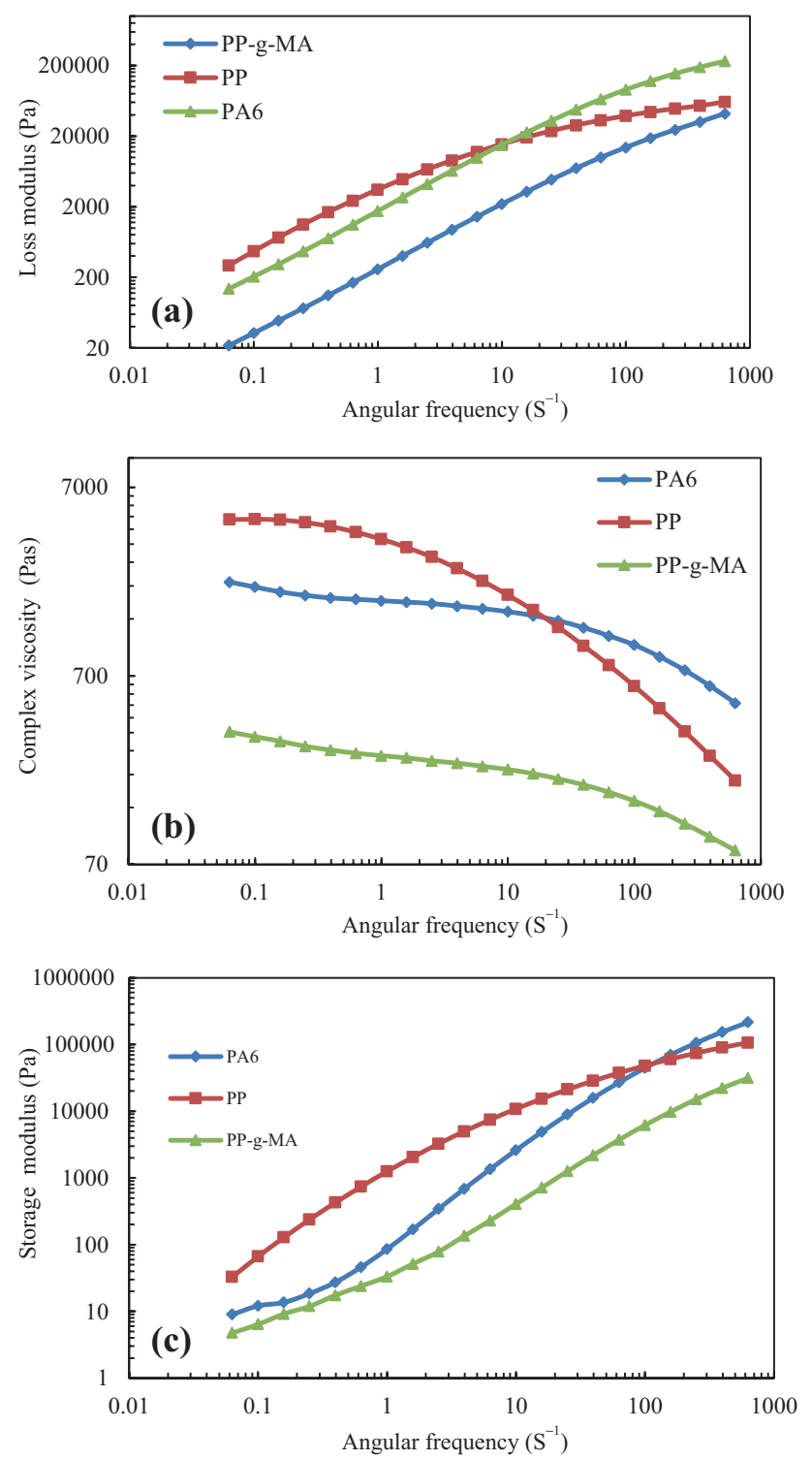

Figure 1. (a) Loss modulus, (b) complex viscosity and (c) storage modulus $v s$. angular frequency for polypropylene, polyamide 6 and pure polypropylene-grafted-maleic anhydride.

Polyamide phase composition effect (reinforced phase) on RMS test results In order to study the effect of polyamide phase composition on microfibrillar composites rheology the RMS and MFI tests were employed and measurements on the prepared samples were conducted. The movability of the PP chains is limited due to the existence of stiff and solid polyamide phase when the samples deform at small strain amplitude. Decrease in the movement of the PP chain means that higher external energy is needed for a specific deformation amount. The friction at the interface of two phases increases with an increase in the polyamide phase, causing further movement restrictions for chains and leading to higher energy loss [11]. Based on this interpretation, a direct connection among the storage modulus, loss modulus and the
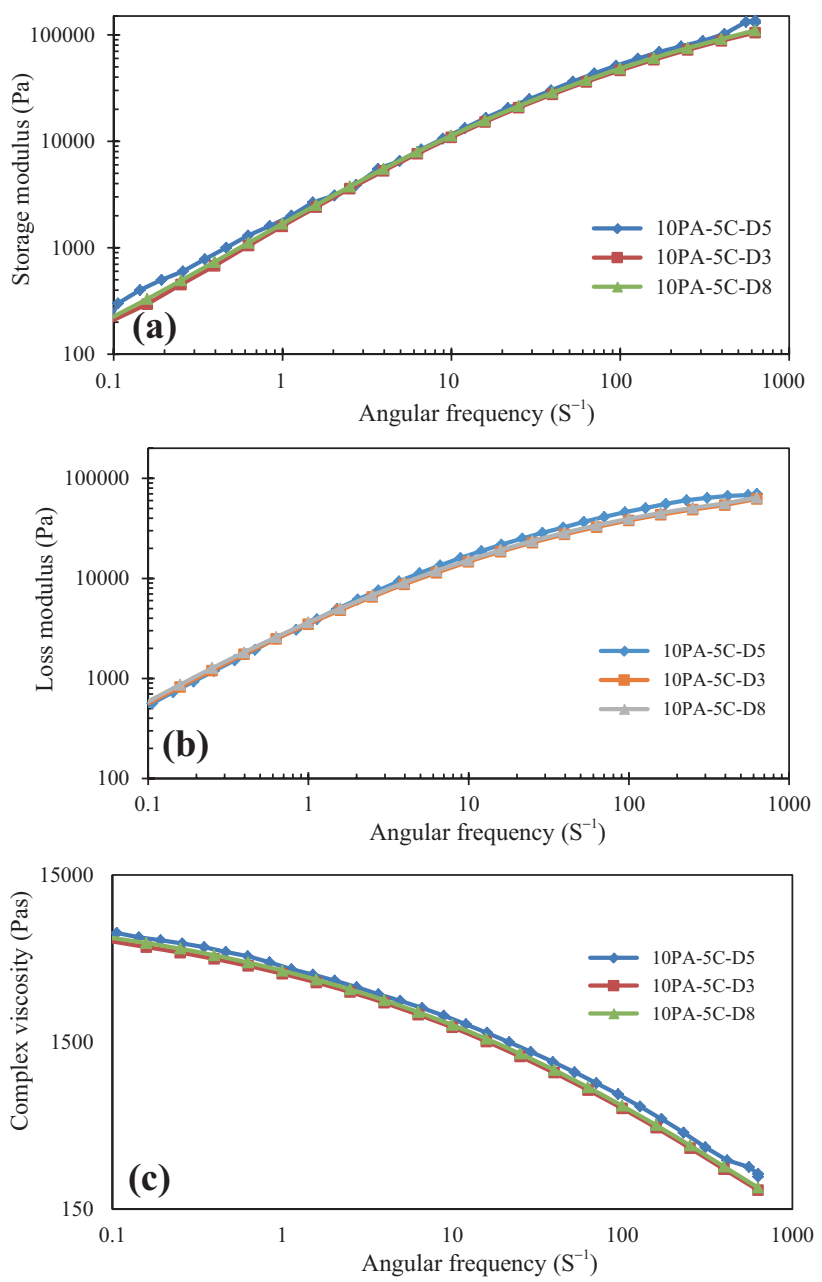

Figure 2. (a) Storage modulus, (b) complex viscosity and (c) loss modulus $v s$. angular frequency for drawing ratios of 3, 5 and 8 .

polyamide phase is evident. Results of storage modulus and loss modulus are shown in figure $3 \mathrm{a}$ and $\mathrm{b}$, respectively.

In figure $3 \mathrm{c}$, the relationship between complex viscosity and angular frequency for microfibrillar composites with different reinforced phase compositions is shown. It is evident that the synthesized composites show desirable pseudoplastic behaviour and encounter shear thinning with increasing angular frequency. The absolute complex viscosity is defined by the below relationship:

$$
\left|\eta^{*}\right|=\sqrt{\left(\frac{G^{\prime}}{\omega}\right)^{2}+\left(\frac{G^{\prime \prime}}{\omega}\right)^{2}}
$$

According to equation (1), complex viscosity variation is mainly a function of the viscoelastic modulus, which is dependent on the morphology and the composition of reinforced phase. As mentioned earlier in this paper, the direct relation of PA6 with both the loss and storage modulus consequently increases the complex viscosity of composites. 

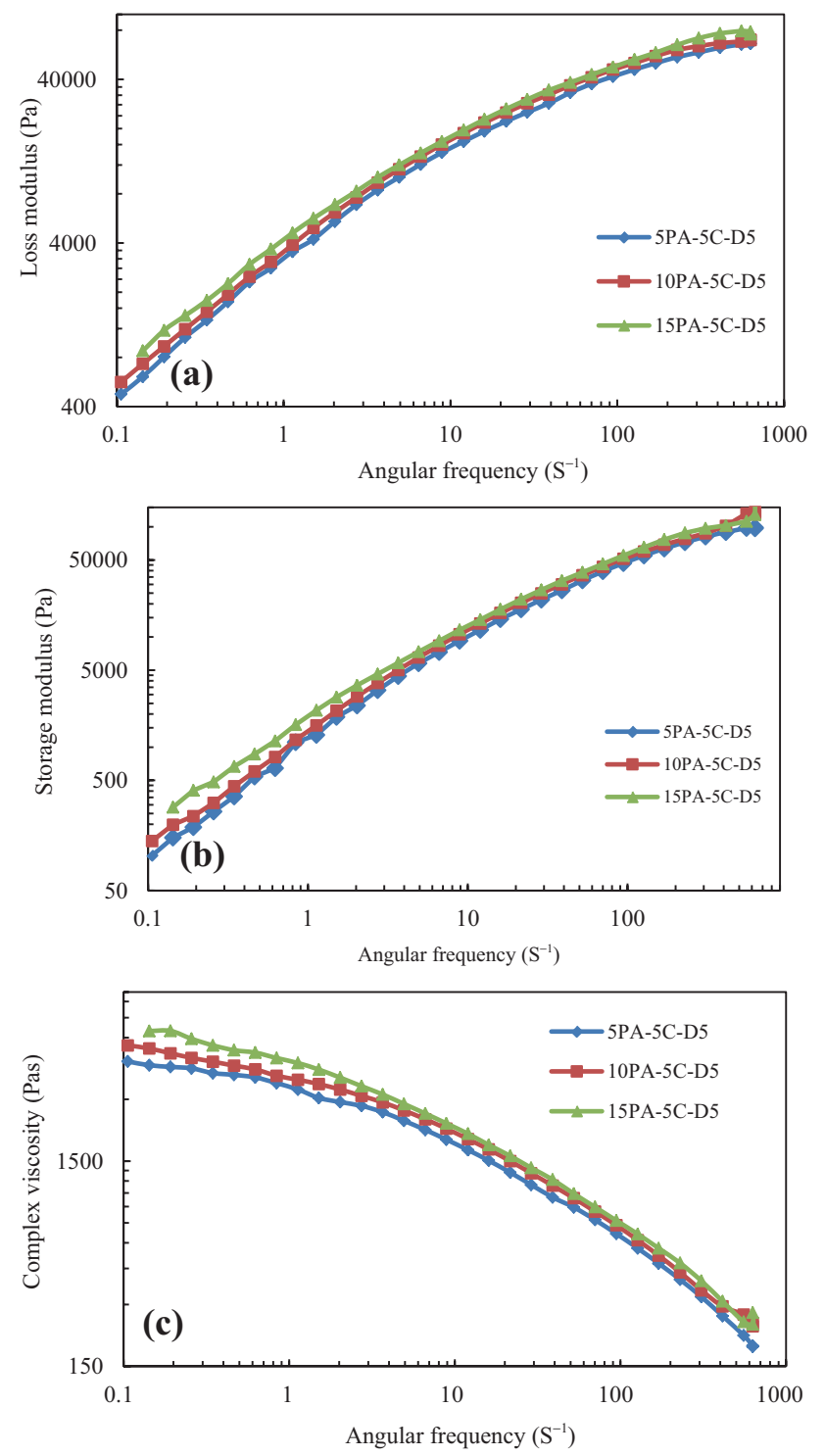

Figure 3. (a) Loss modulus, (b) storage modulus and (c) complex viscosity $v s$. angular frequency of the composite (at drawing ratio 5) for varied composition of polyamide 6 .

Compatibilizer agent effect on RMS test results Figure 4a-c shows the complex viscosity, loss modulus and storage modulus of two samples that are identical in terms of the reinforced phase composition and drawing ratio and different in terms of the absence or presence of compatibilizer. The sample with $5 \mathrm{wt} \%$ compatibilizer shows lower rheological properties than those of the sample with no compatibilizer. The RMS test results for pure materials and microfibrillar composites indicate lower storage modulus, loss modulus and complex viscosity of the sample with PP-g-MA compared with pure $\mathrm{PP}$; the main cause for this rheological property loss is the lesser external energy required for a certain amount of deformation in the sample with compatibilizer compared with the sample with no compatibilizer.
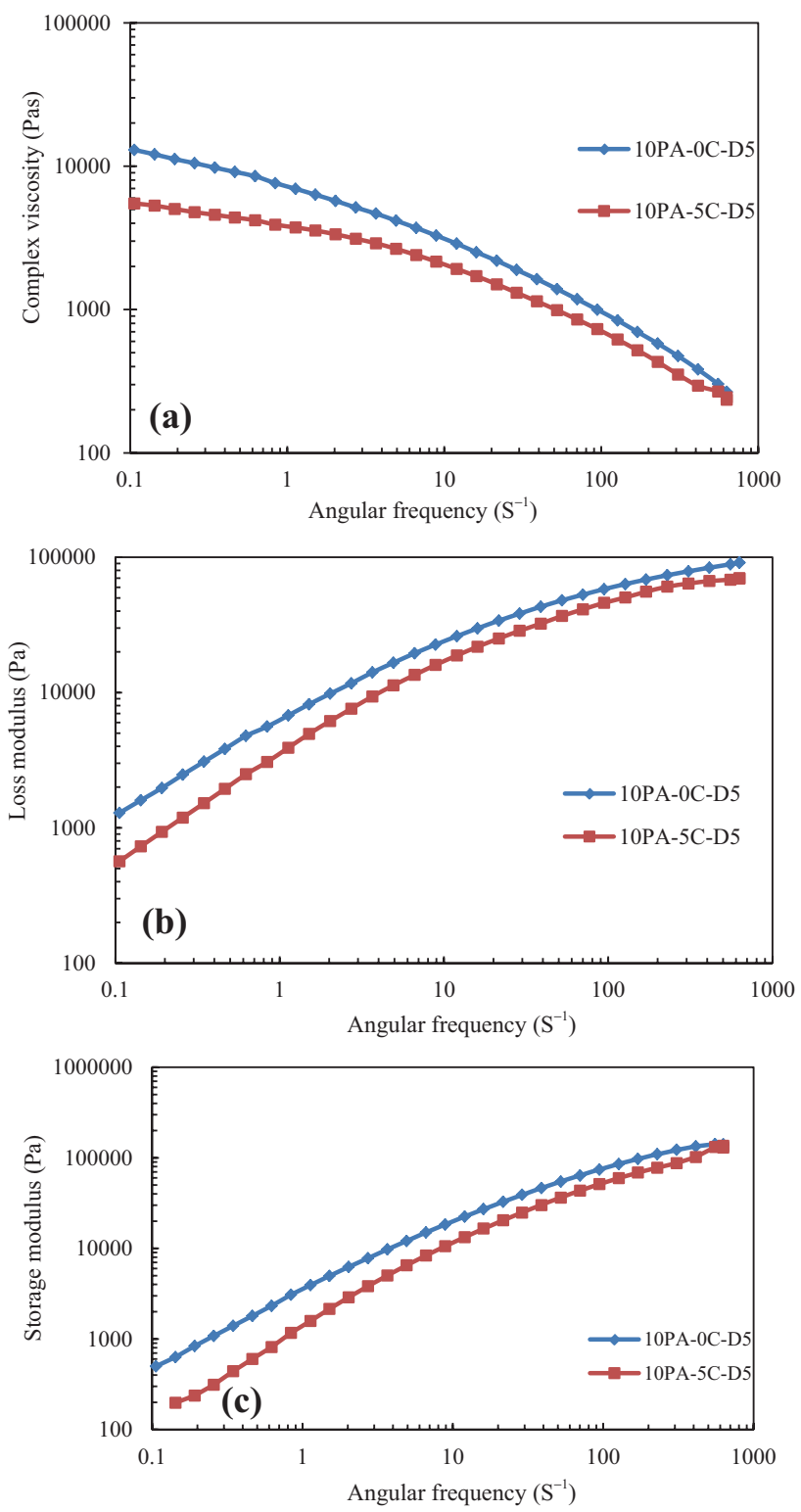

Figure 4. (a) Complex viscosity, (b) loss modulus and (c) storage modulus $v s$. angular frequency of the composite (at drawing ratio 5) in the presence and the absence of compatibilizer.

Microfibrillar structure effect on RMS test results Blends with identical composition but different morphologies were prepared to investigate the effects of microfibrillar structure on the rheology of composites. In the process of preparing blends using droplet-matrix morphology, materials were collected between the interval of the die extruder and the drawing device. Materials had drop-matrix morphology before entering the drawing device; however, they had a fibre-matrix structure after leaving the drawing device.

Figure 5a-c shows the rheological properties obtained from the RMS test for samples with identical composition but difference in morphology (fibre and droplet). As the samples deform in low-strain domains, the movement of the PP chains 

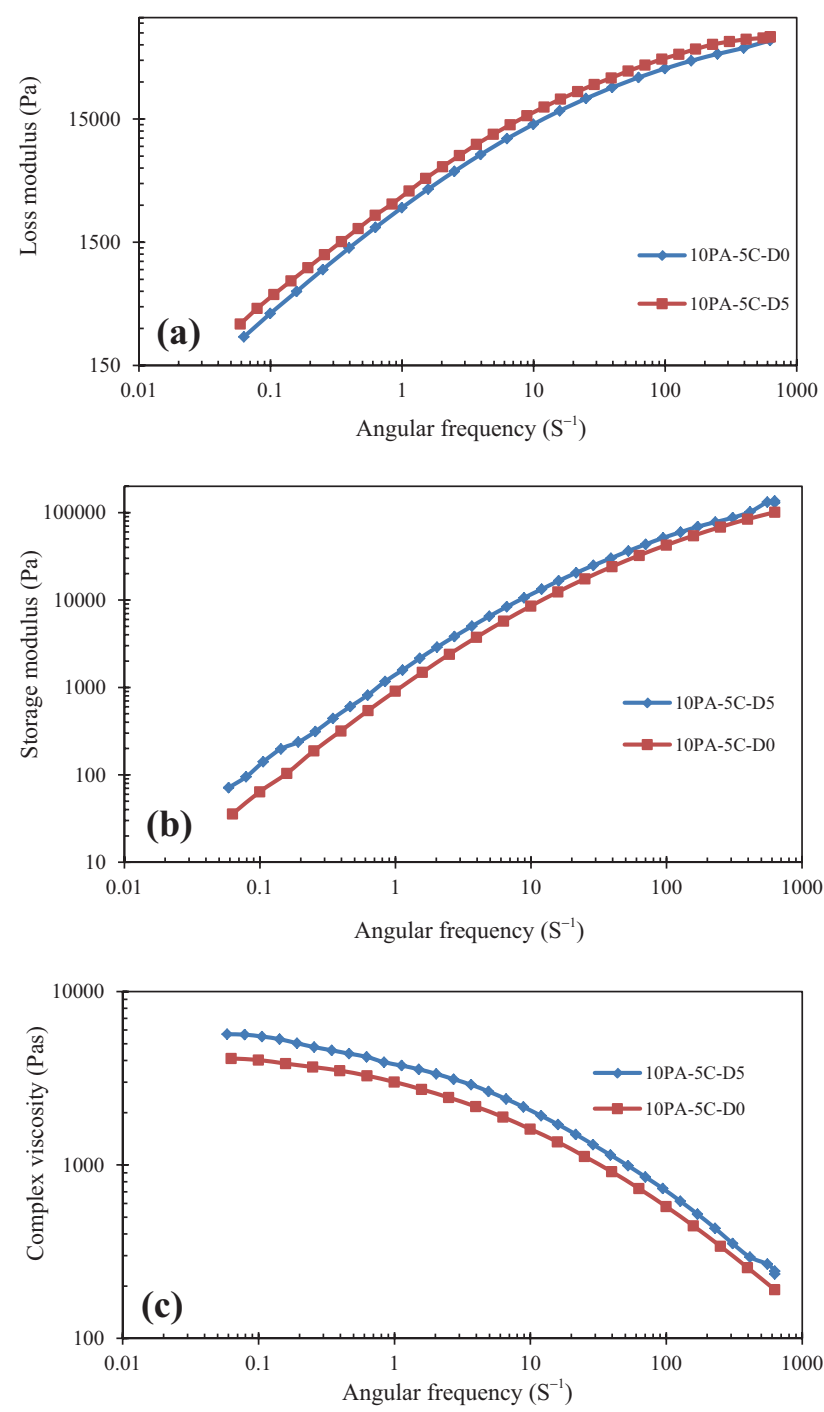

Figure 5. (a) Loss modulus, (b) storage modulus and (c) complex viscosity $v s$. angular frequency of samples with droplet and fibre morphology.

is limited due to existence of stiff and solid polyamide phase, which results in more external energy required for deformation. Regarding the fibre morphology of the polyamide phase and increased movement restrictions for PP chains, friction at the interface of two phases increases and leads to more energy loss compared with droplet morphology. Furthermore, changing the morphology from the droplet matrix to fibre matrix significantly increased storage modulus, loss modulus and complex viscosity.

\section{1b Capillary rheometer test:}

Compatibilizer effect on the capillary rheometer results: A capillary rheometer was used to determine the rheological behaviour of microfibrillar composites at high shear rates. Tests were performed at a temperature of $210^{\circ} \mathrm{C}$ and the piston speed of $0.2,0.6,2.0,6.0$ and $20.0\left(\mathrm{~cm} \mathrm{~min}^{-1}\right)$. The
Rabinowitsch equation was used to express the results. The real shear rate on the walls is obtained by the equation

$$
\gamma_{w}^{\circ}=\left(\frac{3 n^{\prime}+1}{4 n^{\prime}}\right) \gamma_{a}^{\circ}
$$

The parameter $n^{\prime}$, which is a criterion for the pseudoplastic behaviour of polymers, was calculated from the slope of logarithmic diagram of stress on the wall vs. the apparent shear rate. The methods for calculating $n^{\prime}$ are shown in figure $6 \mathrm{a}$ and $\mathrm{b}$ for samples without compatibilizer and samples with compatibilizer, respectively.

According to the obtained values of $n^{\prime}$ for samples, it can be concluded that the viscosity in the samples containing compatibilizer is higher compared with the samples with no compatibilizer (see figure $6 \mathrm{c}$ ); as a result they are more processable due to their pseudoplastic behaviour. The values of $n^{\prime}$ obtained from the capillary rheometer test for samples with and without compatibilizer are 0.3828 and 0.4365 , respectively.
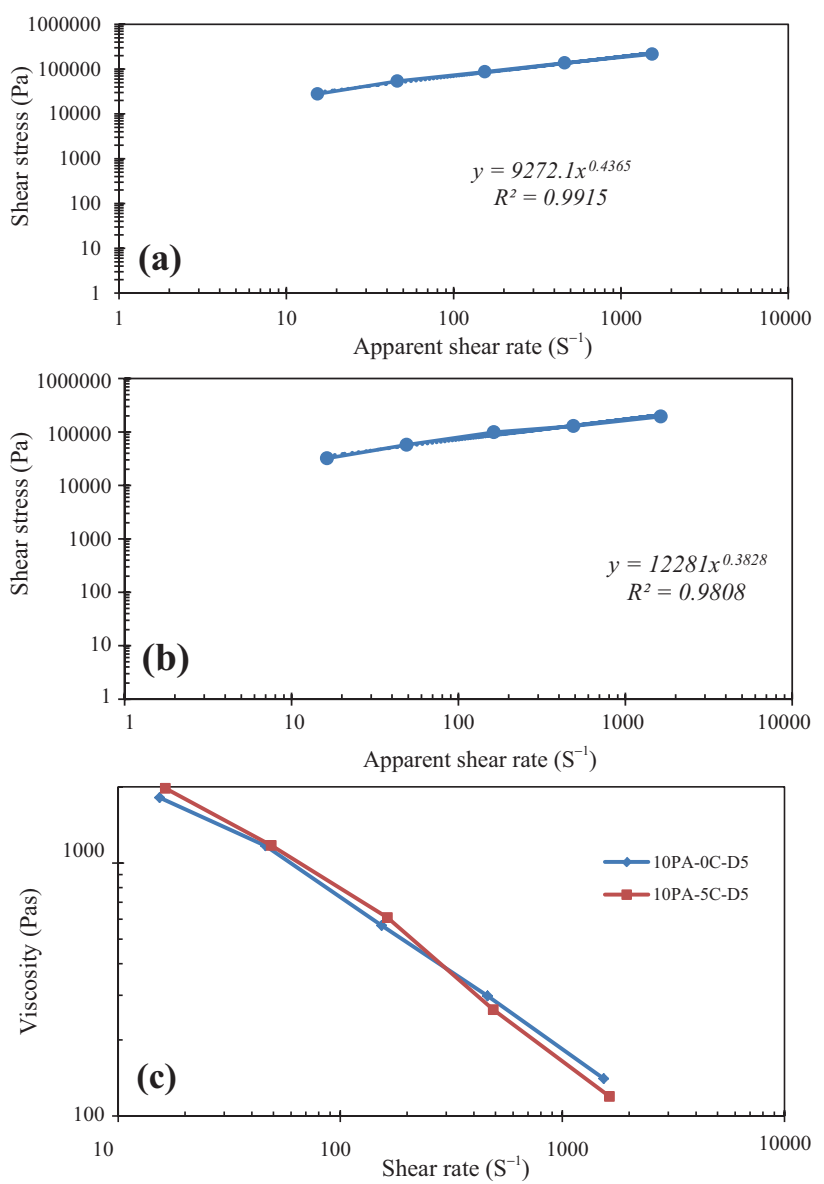

Figure 6. Shear stress vs. apparent shear rate for (a) sample without compatibilizer and (b) with compatibilizer; (c) viscosity-shear rate for the composite in the presence and the absence of compatibilizer. 

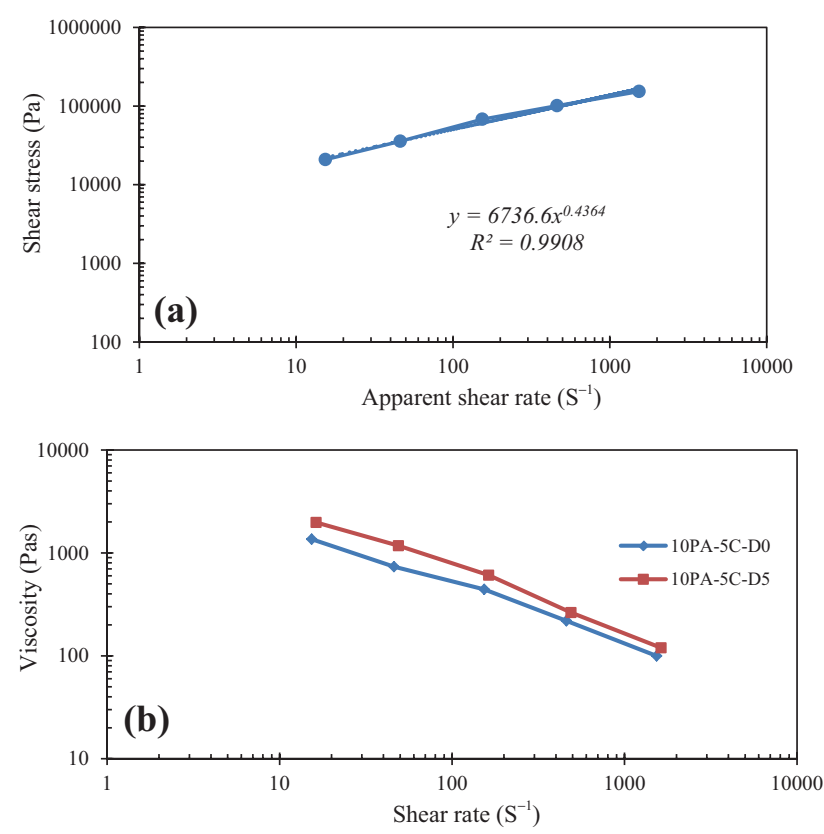

Figure 7. (a) Shear stress vs. apparent shear rate for sample with droplet morphology. (b) Viscosity $v s$. shear rate curve for samples with fibre and droplet morphology.

Microfibrillar structure effect on capillary rheometer results: In order to examine the relationship between rheology and morphology of the microfibrillar composite, the capillary rheometer test (at a temperature of $210^{\circ} \mathrm{C}$ and the piston speed of the $0.2,0.6,2.0,6.0$ and $\left.20\left(\mathrm{~cm} \mathrm{~min}^{-1}\right)\right)$ was carried out on samples with identical compositions but different morphology types. Calculated values of $n^{\prime}$ parameter for samples with fibre and droplet morphology are shown in figures $6 \mathrm{~b}$ and $7 \mathrm{a}$.

Then, the real shear rate was calculated using the Rabinowitsch equation. The viscosity-shear rate diagram of samples with identical components composition but different morphologies is shown in figure $7 \mathrm{~b}$. As is evident, the behaviour of the samples is desirably pseudoplastic. Furthermore, the results for high shear rates are consistent with the results of the RMS test. For same shear rates, samples with fibre morphology have a higher apparent viscosity than samples with droplet morphology. The polyamide fibres increase interface with PP matrix and the friction increases consequently, resulting in flowability reduction and increase in the viscosity compared with samples with droplet morphology.

3.1c MFI test results: The effects of variable levels of drawing ratio, the reinforced phase composition (PA6) and the compatibilizer composition on the rheological properties were studied by the MFI test.

In figure 8a, changes in MFI of microfibrillar composites prepared at drawing ratios of 3,5 and 8 are shown. The test was carried out at a temperature of $210^{\circ} \mathrm{C}$, with $2.160 \mathrm{~kg}$ weight. Two main factors that affect the MFI of microfibrillar composites are their entanglement and orientation [13]. It is observed that the MFI of microfibrillar composites declined on increasing the drawing ratio from 3 to 5 , due to increase in fibre entanglement. However, the micro-fibres are oriented in the draw direction at higher drawing ratios, and therefore, they show less resistance to deformation. In the latter case, the orientation of microfibrillar composites toward the flow path overcomes their entanglement, resulting in an increase in the MFI.

Figure $8 \mathrm{~b}$ shows the changes in MFI attributed to different reinforced phase compositions. MFI was measured at $210^{\circ} \mathrm{C}$ with 2.160 and $5 \mathrm{~kg}$ weights. As can be seen in figure $8 \mathrm{~b}$, higher polyamide content decreases the MFI. The polyamide composition increase and formation of fibre morphology lead to entanglement of micro-fibres in PP and deviation of MFI from its normal value [13]. On changing the weights from 2.160 to $5 \mathrm{~kg}$ and increasing the stress on the polymer, MFI increased and melt viscosity decreased, which indicate pseudoplastic behaviour of the samples.

Figure 8c shows the MFI of samples at a drawing ratio of 5 , equal PA6 composition and different percentages of the compatibilizer. It is observed that due to the high MFI of PP-g-MA (80 g/10 min), adding compatibilizer has a direct influence on the MFI of composites.

The MFI of samples with fibre and droplet morphologies is shown in figure 8d. The composite samples with fibre morphology were prepared at a drawing ratio of 5 . Tests were carried out at a temperature of $210^{\circ} \mathrm{C}$ with $2.160 \mathrm{~kg}$ weight. It can be observed that changing the morphology from droplet to fibre morphology has led to an abrupt drop in MFI. The fibre morphology caused entanglement of micro-fibres in PP chains, which explains the MFI decrease compared with the normal state. The restriction of movement in the chains of PP in the fibre morphology causes reduction in flowability and thus reduces the MFI.

\subsection{Morphological result}

The scanning electron microscopy (SEM) test was used to evaluate the effect of reinforced phase composition, compatibilizer composition and the drawing ratio on the morphology and diameter of the fibres in the synthesized microfibrillar composites.

3.2a Reinforced phase composition effect on the morphology of microfibrillar composites: Figure 9 shows the SEM images of microfibrillar composites produced at a drawing ratio of 5, containing $5 \mathrm{wt} \%$ of compatibilizer, and various reinforced phase compositions. The images were taken after the extraction of the matrix phase (PP) in boiling xylene. The average diameter of micro-fibres for 5PA-5C-D5, 10PA-5C-D5 and 15PA-5C-D5 samples was 0.15, 0.18 and $0.19 \mu \mathrm{m}$, respectively. According to values obtained, it can 

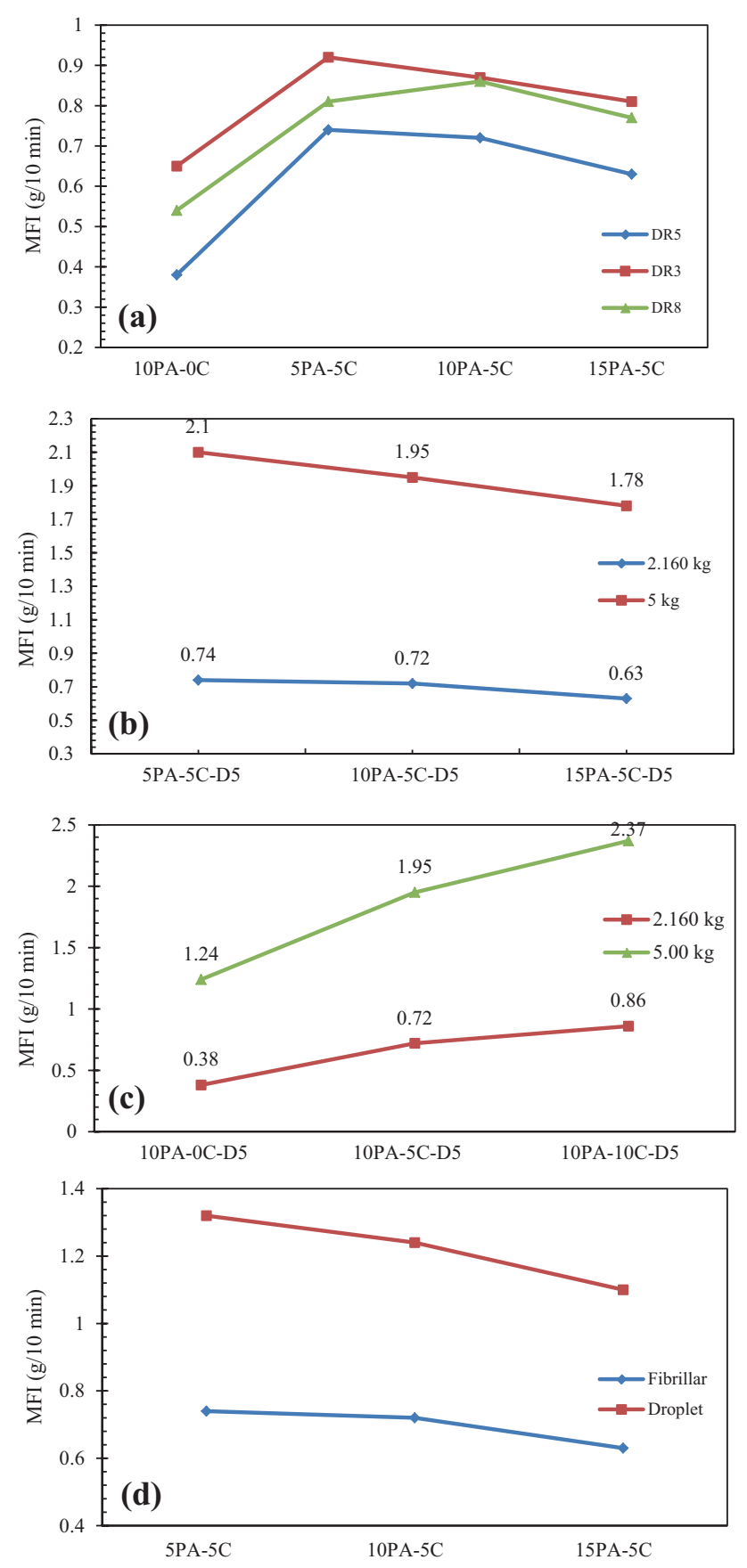

Figure 8. Melt flow index of composites (a) at drawing ratios of 3,5 and 8, (b) the polyamide 6 composition and the amount of compatibilizer (at drawing ratio 5), (c) at a drawing ratio of 5, equal PA6 composition and different percentages of the compatibilizer and (d) for fibre and droplet morphologies (at drawing ratio 5).

be inferred that the average diameter of microfibrillar composites increases with an increase in the reinforced phase composition. SEM image of sample 10PA-5C-D5 indicates the formation of a network of entangled fibres of polyamide phase in the matrix. 3.2b Compatibilizer content effect on the morphology of microfibrillar composites: SEM images of microfibrillar composites with the absence and the presence of the compatibilizer were obtained to study the performance and effect of the compatibilizer agent on the morphological properties of the composites (figure 10).

The fibre diameter for samples containing the compatibilizer is lower compared with the samples with no compatibilizer. Thus, the compatibilizer addition led to thinner and more uniformly distributed polyamide fibres. Moreover, undeformed droplets in the samples containing compatibilizer are much less than in the samples without the compatibilizer.

\section{2c Draw ratio effect on the morphology of microfibrillar} composites: In order to investigate the effect of the drawing ratio on the morphology of microfibrillar composites, samples with identical polyamides and compatibilizer composition but different drawing ratios were prepared. Figure 11 shows the SEM images of composites synthesized at drawing ratios of $0,3,5$ and 8 . To obtain the SEM image of the fracture surface of blends prepared at different drawing ratios, the blends were inundated in liquid nitrogen and broken afterwards. The SEM images were taken after the extraction of the matrix phase (PP) in boiling xylene. The average diameters of micro-fibres for 10PA-5C-D3, 10PA-5C-D5 and 10PA-5C-D8 samples were $12.86,0.18$ and $3.29 \mu \mathrm{m}$, respectively. From the SEM images and average diameter of the micro-fibres, it can be inferred that composites prepared at a drawing ratio of 5 has the smallest diameter and therefore microfibre entanglement at a drawing ratio of 5 is more compared wsith drawing ratios of 3 and 8 . The results from the SEM test (morphology) had acceptable agreement and are consistent with rheological MFI measurements and RMS test results.

\subsection{Mechanical properties results}

The mechanical properties of the composites include tensile strength, elongation at break and Young's modulus, which will be evaluated separately in this study under the influence of factors such as different reinforcing phases, draw ratios and compatibilizer.

\section{3a Reinforced phase composition effect on the mechanical} properties of microfibrillar composites: Figure 12a shows the tensile strength graph of microfibrillar composite samples prepared at a draw ratio of 5 . The samples 10PA-0C-D5 and 10PA-5C-D5 are identical in terms of the polyamide phase composition and the draw ratio, but different in terms of absence or presence of compatibilizer. The compatibilizer bonds between PA6 and PP micro-fibres, which results in improved cohesion and, subsequently, transmission of more tension from the matrix to the fibres; this would increase the tensile strength. For samples containing the compatibilizer, increase in the PA6 composition initially caused tensile strength decrease; however, later it caused increase as the test was carried out. What can be interpreted from this is 

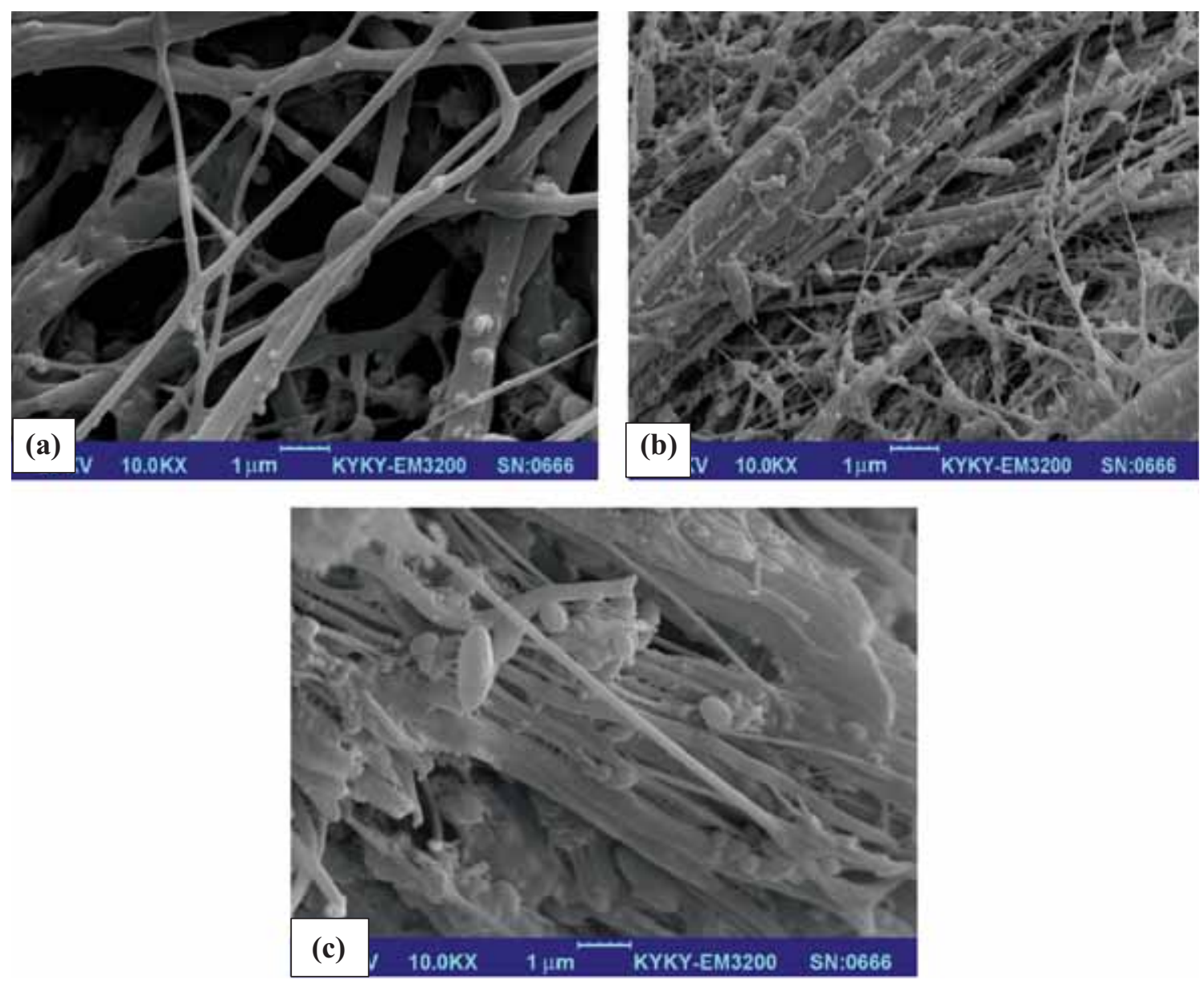

Figure 9. SEM images of samples: (a) 5PA-5C-D5, (b) 10PA-5C-D5 and (c) 15PA-5C-D5.
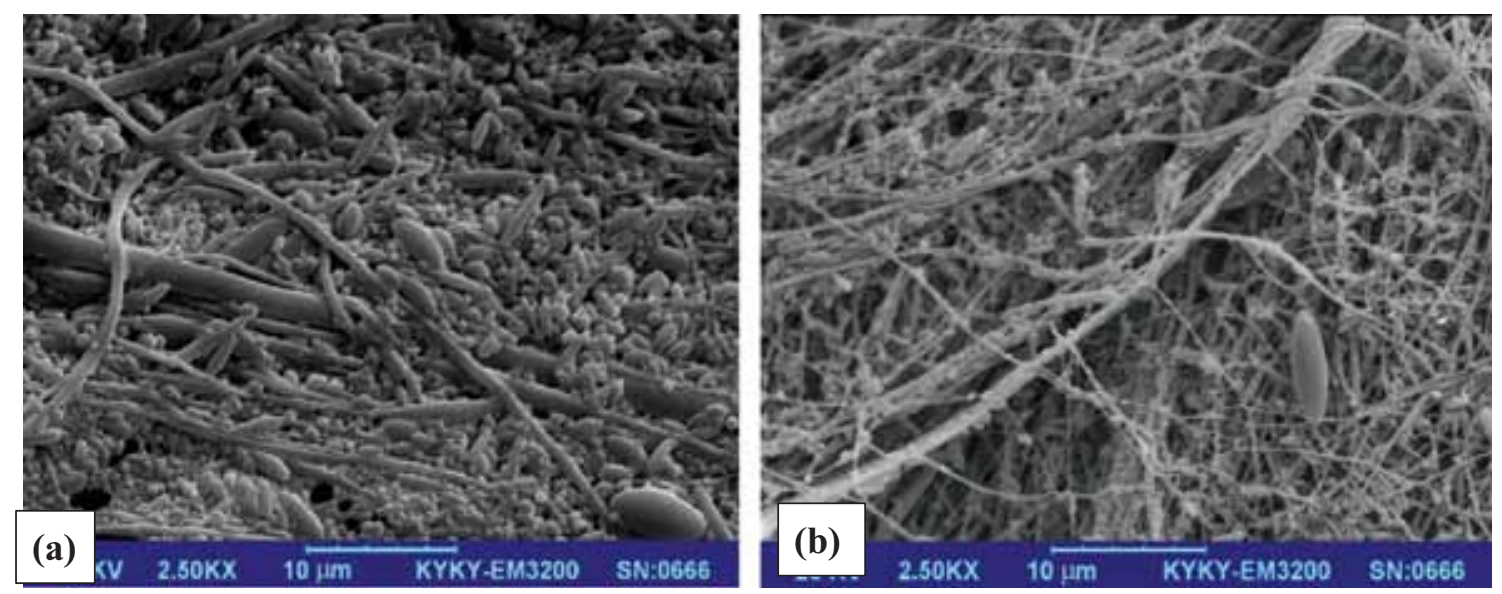

Figure 10. Effect of the compatibilizer on morphology: (a) 10PA-0C-D5 and (b) 10PA-5C-D5.

that high level of fibres is accompanied with loss of their reinforcement role and the matrix is not able to cover the gap between the fibres [5]. It can be said that short fibres lead to strength increase until a certain weight percentthe optimum percentage. This implies that on surpassing this amount, fibres act as a filler and reduce the composite strength.
The same trend was observed for other composites prepared in this work.

A diagram of Young's modulus $v s$. the reinforcing phase for composites prepared at a draw ratio of 5 is shown in figure $12 \mathrm{~b}$. It can be observed that addition of polyamide to the PP matrix increased Young's modulus compared with pure PP. This can 

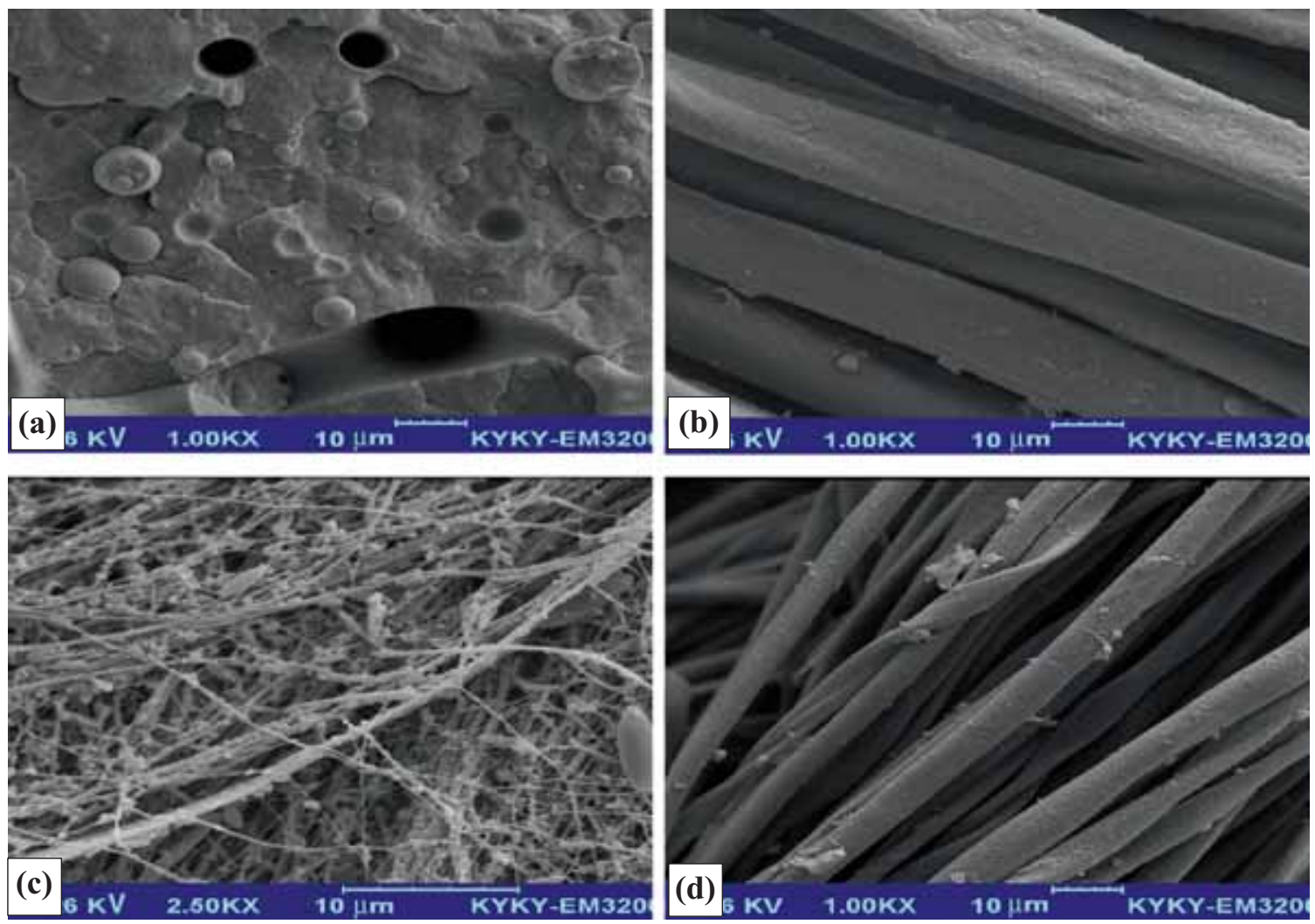

Figure 11. Effects of drawing ratio on morphology: (a) 10PA-5C-D0, (b) 10PA5C-D3，(c) 10PA-5C-D5 and (d) 10PA-5C-D8.

be due to higher bulk modulus of polyamide than that of PP. At the low composition of reinforcing phase, the fibres are not sufficiently present in the matrix to protect the matrix against composite deformation, which results in non-uniform distribution of composite stress and stress concentration. However stress distributes more uniformly and different parts of the composite are strengthened uniformly if the reinforcing phase composition is increased; as a result, Young's modulus and hardness of the composite increase accordingly. Samples containing the compatibilizer have higher Young's modulus compared with samples without the compatibilizer, which is due to good adhesion between the matrix and reinforcement phase, the polyamide reinforcing effect and high ratio of length to diameter of micro-fibres. Moreover adding fibres to the matrix increases the composite's crystallinity, and thus Young's modulus increases.

The composite's elongation at break achieved from the tensile property test is an important factor in enlightening the concept of composite toughness. Elongation at break diagram for composites made at a draw ratio of $5 \mathrm{vs}$. the reinforcement phase composition is shown in Figure 12c. It can be observed that with increasing percentage of polyamide micro-fibres, the incompatibility between these two immiscible polymers is increased. Increase in incompatibility leads to more cracks and thereby reduces the elongation at break of the composite. Applying stress in composites without the compatibilizer causes small cracks at the interface of two phases due to insufficient adherence between the matrix and micro-fibres; therefore, these cracks cause lower elongation at break in composites without the compatibilizer.

3.3b Draw ratio effect on the mechanical properties of microfibrillar composites: Figure 13a and b shows the effect of draw ratio on tensile strength and Young's modulus of prepared composites. The produced composites were identical in the percentage of the matrix phase and reinforcement phase composition, but were different in the draw ratio. It is evident that with increase in the draw ratio, tensile strength and Young's modulus initially increase and then decrease. The composite sample made at a draw ratio of 8 has a tensile strength and Young's modulus lower than those of the sample made at a draw ratio of 5 . It can be concluded that the tensile strength and Young's modulus of microfibrillar composites increase with increasing draw ratio until they reach their maximum value between draw ratios of 5 and 8 , and then decrease. The reason for lower tensile strength and Young's modulus of those composites made at a draw ratio of 3 can be the high average diameter of the polyamide micro-fibres and, as a result, lower dimensional ratio. According to $\mathrm{Li}$ et al [15], increasing the draw ratio increases the fragility of the composite. 

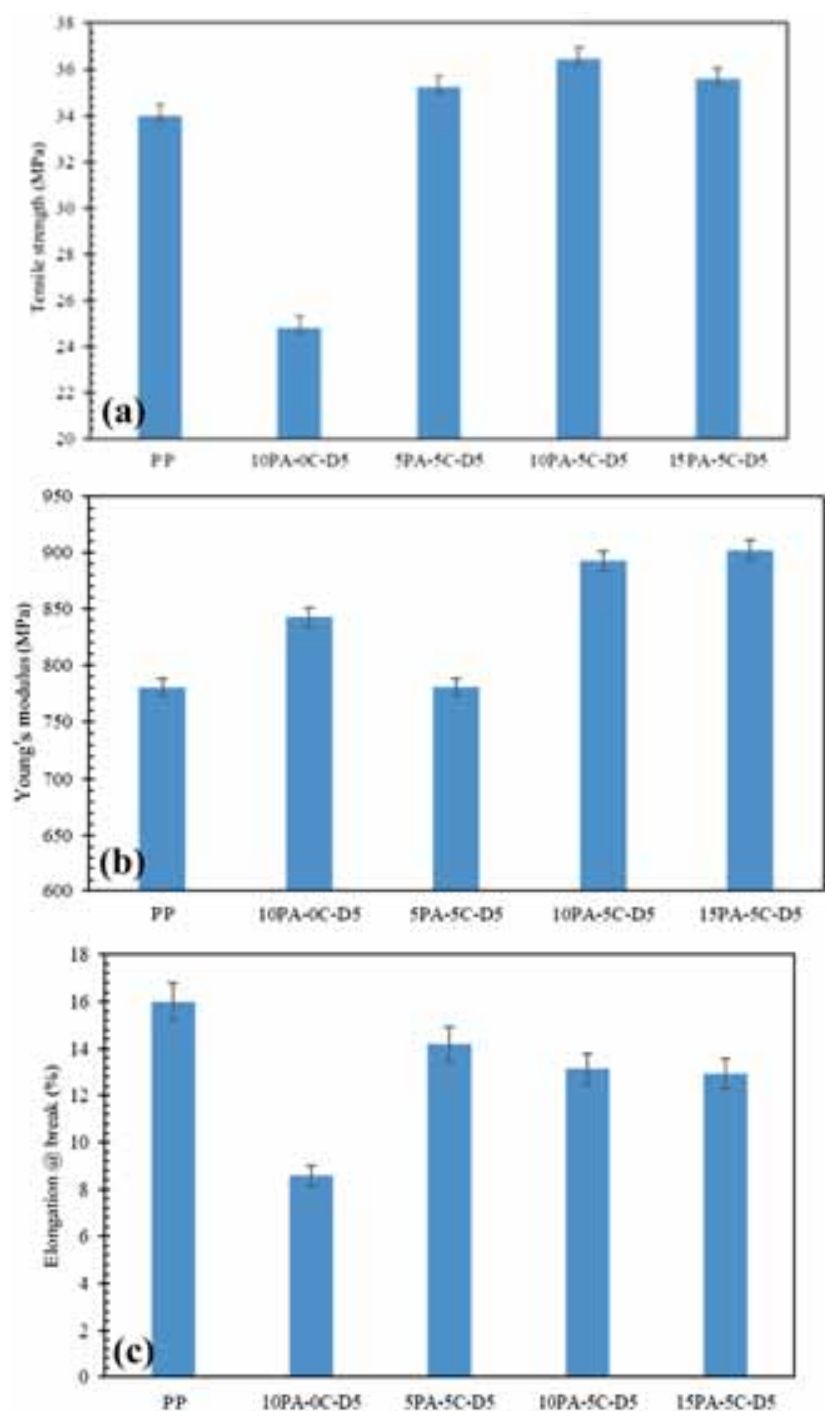

Figure 12. Reinforced phase composition effect on the mechanical properties of microfibrillar composites: (a) tensile strength, (b) Young's modulus and (c) elongation at break.

Figure 13c shows the changes in elongation at break of composites prepared at draw ratios of 3,5 and 8 . It can be seen that on increasing the draw ratios from 3 to 5 , stress transmission occurs due to improved length of polyamide micro-fibres. Better transmission of stress causes an increase in toughness of the composites; as a result of this toughness, the elongation at break, which is a measure of the toughness of the composites, increases accordingly. Moreover, elongation at break of composites is decreased on increasing the draw ratio from 5 to 8. According to Li et al [15], increasing the draw ratio by a certain amount causes fragility in the materials. Therefore decrease in elongation at break of composites made at a draw ratio of 8 is evident. The conclusion inferred is as follows: increase in draw ratio changes the behaviour of the composites from brittle to tough and again changes it to the fragile state.
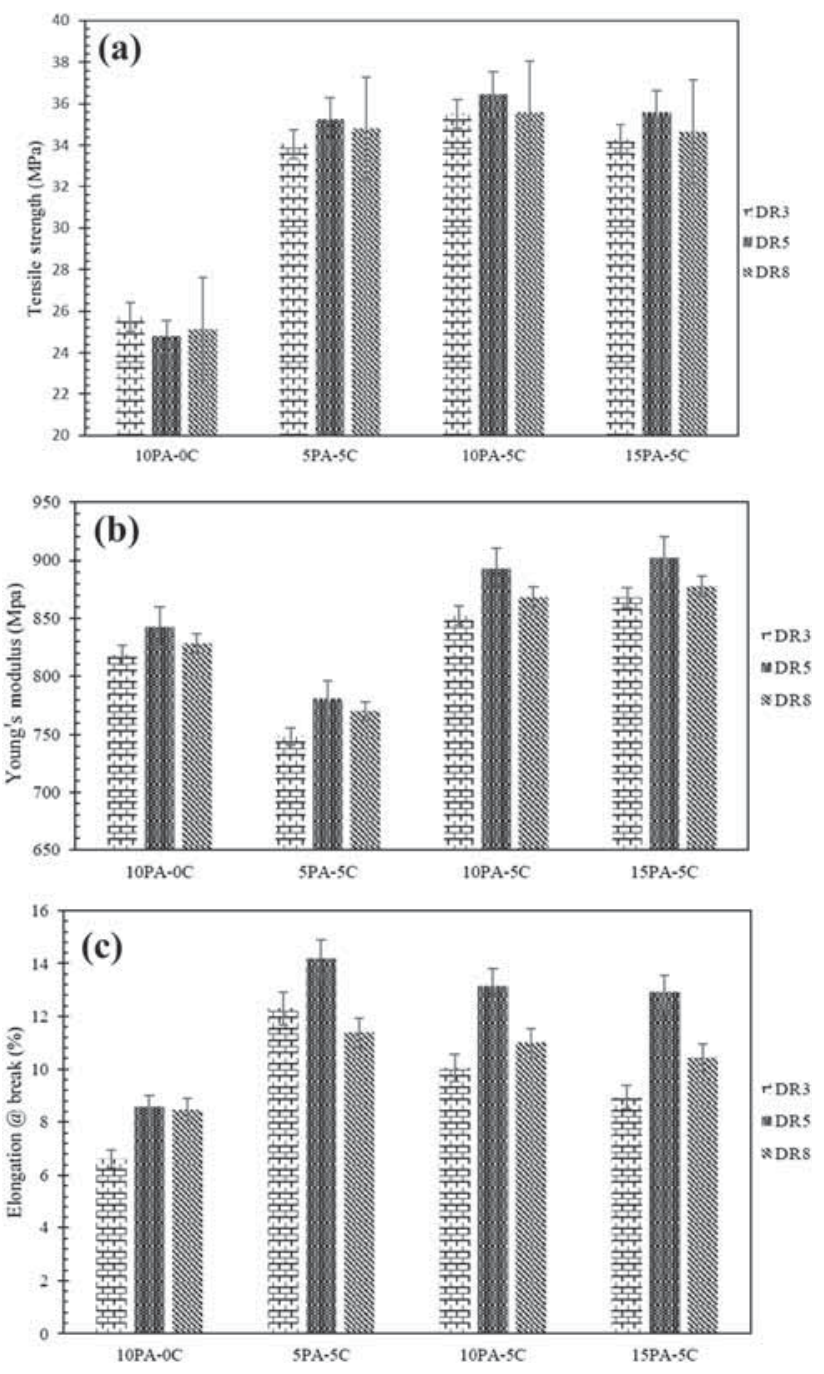

Figure 13. Draw ratio effect on the mechanical properties of microfibrillar composites: (a) tensile strength, (b) Young's modulus and (c) elongation at break.

3.3c Compatibilizer content effect on the mechanical properties of microfibrillar composites: In order to evaluate the effect of the compatibilizer consistency on the mechanical properties, samples with identical reinforcing phase composition (10\% PA6) and draw ratio of 5 were prepared. The compatibilizer amounts used in the samples were 0,5 and $10 \%$. Tensile strength, elongation at break and Young's modulus of these composites are studied in the following. Figure 14a shows the results of tensile strength of the microfibrillar composites prepared with $10 \mathrm{wt} \%$ of reinforcing phase at the draw ratio of 5. The compatibilizer improves the adherence between the phases and as a result, more transmission of stress occurs from the matrix of fibres, which is due to creation of bonds between the PA6 microfibres and PP. The mentioned reasons lead to increase in the tensile strength (47\%) of the composite on adding 5\% of the compatibilizer. However, increasing the amount of 
compatibilizer from 5 to $10 \%$ decreases the tensile strength. The compatibilizer PP-g-MA causes reduction in the tensile strength, which is due to its low molecular weight and weak mechanical strength at the interface between the phases, which is the main reason behind the decrease in the tensile stress with the increase in the amount of the compatibilizer.

Figure $14 \mathrm{~b}$ shows the elongation at break for composites prepared at a draw ratio of 5 and with different amounts of compatibilizer. In the sample without compatibilizer, some cracks appear at the interface of two phases on applying stress due to incompatibility and poor adhesion between the matrix and micro-fibres. The growth of these micro-cracks causes lower elongation at break and reduces the toughness of the composite consequently. Adding $5 \mathrm{wt} \%$ of compatibilizer improves the interface, enhances adhesion between the phases and the length to diameter ratio of micro-fibres. The occurrence of these events increases the stress transmission from the matrix to micro-fibres and thereby increases elongation
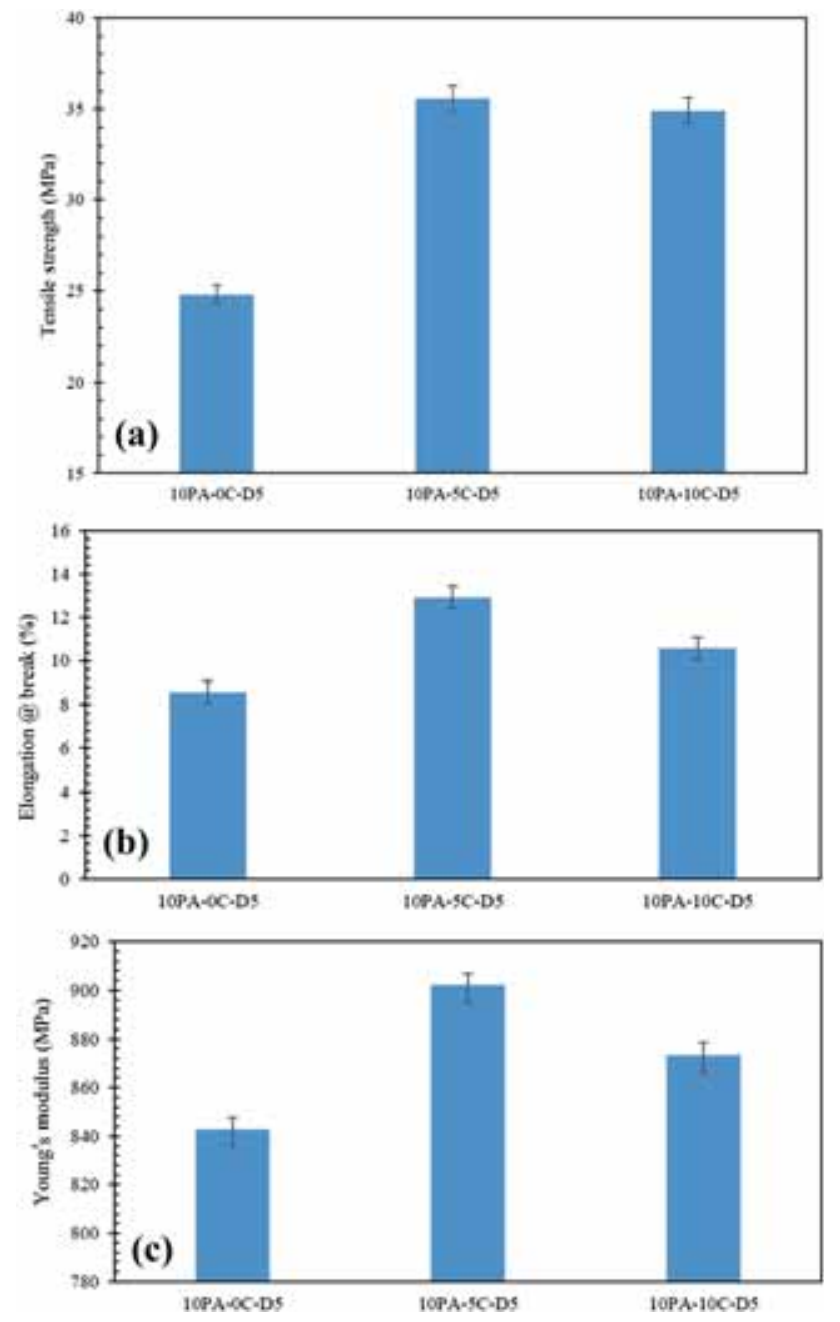

Figure 14. Compatibilizer content effect on the mechanical properties of microfibrillar composites: (a) tensile strength, (b) elongation at break and (c) Young's modulus. at break of the composite. However, increasing the amount of compatibilizer to $10 \mathrm{wt} \%$ decreases the failure strength of the composite due to stress concentration at the interface of phases and also due to poor mechanical properties of the compatibilizer, which are related to its low molecular weight.

Young's modulus or tensile modulus is defined as the initial slope of the stress-strain diagram, which is usually considered at strain less than $2 \%$. The Young's modulus represents the resistance of a piece to deformation and depends on factors such as the mechanical properties of fibres and matrix, and the type of interface between them. In figure 14c, Young's modulus of samples with the same composition of the reinforcing material but different amounts of compatibilizer is shown. As observed, Young's modulus exacerbates on adding $5 \mathrm{wt} \%$ of the compatibilizer due to fair adhesion between the matrix and reinforcing phase, high ratio of length to diameter and high reinforcement effect of polyamide micro-fibres [16]. However, on increasing the amount of compatibilizer to $10 \mathrm{wt} \%$, a drop in Young's modulus occurs. This could be related to the nature of the compatibilizer, which has poor mechanical properties associated with its low molecular weight.

\section{Conclusion}

In this study, rheology and morphology of the microfibrillar composites synthesized based on reinforced PP-PA6 micro-fibres was assessed. The results of the RMS, rheological measurements and MFI tests showed that the PA6 composition has direct effect on the storage modulus, loss modulus and complex viscosity of microfibrillar composites, while it has a reverse effect on the MFI. On increasing the drawing ratio from 3 to 5 , increase in rheological property and decrease in MFI were observed. However, on further increasing the drawing ratio from 5 to 8 , the storage modulus, loss modulus and complex viscosity reduced. In order to investigate the effect of the compatibilizer on the rheological properties of microfibrillar composites, the RMS, the MFI and the capillary rheometer tests were performed on samples in the absence and the presence of compatibilizer agent. The results showed that the presence of the compatibilizer reduced the rheological properties of the samples while it increased the MFI. The results of mechanical tests on the samples made at different drawing ratios showed that on increasing the ratio from 3 to 5 , the mechanical properties of composites (tensile strength, Young's modulus and elongation at breakage) increase. However, on increasing the ratio from 5 to 8 , reduction in the mechanical properties was observed. On increasing the amount of the polyamide phase, tensile strength of compatibilized composites increased initially, but decreased at higher composition of polyamide phase; this increase is due to intensified defects in the composite structure. The application of $5 \mathrm{wt} \%$ of the compatibilizer led to an increase in the mechanical properties of composites compared with incompatible samples. Nevertheless, on 
increasing the compatibilizer amount to $10 \mathrm{wt} \%$, the mechanical properties decreased due to poor mechanical properties of PP-g-MA. SEM images revealed that the presence of compatibilizer in the microfibrillar composites reduces the number of undeformed drops; moreover, the presence of compatibilizer reduces the average diameter of micro-fibres. On increasing the drawing ratio from 3 to 5 , the average diameter of micro-fibres decreased dramatically; however, on increasing the draw ratio from 5 to 8 , the average diameter of microfibrillar composites increased. Furthermore, the SEM images showed that on increasing the composition of PA6 phase in the composite phase, the mean diameter of micro-fibres increases.

\section{References}

[1] Bhattacharyya D and Fakirov S 2012 Synthetic polymerpolymer composites (Carl Hanser Verlag GmbH Co KG)
[2] Fakirov S and Bhattacharyya D 2015 Engineering biopolymers: homopolymers, blends, and composites (Carl Hanser Verlag GmbH Co KG)

[3] Yi X et al 2010 Eur. Polym. J. 46719

[4] Bigdeli A et al 2012 J. Polym. Res. 191

[5] Chomat D et al 2015 J. Appl. Polym. Sci. 101002

[6] Huang W Y et al 2003 Polym. Int. 521131

[7] Jayanarayanan K et al 2009 Eur. Polym. J. 451738

[8] Li X et al 1997 Polym. J. 29975

[9] Naficy S and Garmabi H 2007 Compos. Sci. Technol. 673233

[10] Pesneau I et al 2002 Polym. Eng. Sci. 421990

[11] Shen J et al 2011 Polym. Adv. Technol. 22237

[12] Tavanaie M, Shoushtari A and Goharpey F 2010 J. Macromol. Sci. B: Phys. 49163

[13] Xu H S et al 2004 Macromol. Mater. Eng. 2891087

[14] Zoldan J, Siegmann A and Narkis M 2005 J. Macromol. Sci. B: Phys. $\mathbf{4 4} 495$

[15] Li Z et al 2002 Polym.-Plast. Technol. Eng. 4119

[16] Houshyar S, Shanks R and Hodzic A 2005 J. Appl. Polym. Sci. 962260 